\title{
Volume targeted versus pressure support non-invasive ventilation in patients with super obesity and chronic respiratory failure: a randomised controlled trial
}

\author{
Patrick Brian Murphy, ${ }^{1,2}$ Craig Davidson, ${ }^{2}$ Matthew David Hind, ${ }^{3}$ Anita Simonds, ${ }^{3}$ \\ Adrian J Williams, ${ }^{2}$ Nicholas S Hopkinson, ${ }^{3}$ John Moxham, ${ }^{1}$ Michael Polkey, ${ }^{3}$ \\ Nicholas Hart ${ }^{4}$
}

\begin{abstract}
- Additional materials are published online only. To view these files please visit the journal online (http://thorax.bmj. com/content/67/8.toc)
\end{abstract}

${ }^{1}$ Division of Asthma, Allergy and Lung Biology, Department of Asthma, Allergy and Respiratory Science, King's College London, London, UK

${ }^{2}$ Lane Fox Translational Respiratory Research Unit, Guy's and St Thomas' NHS Foundation Trust, London, UK ${ }^{3}$ National Institute for Health Research Respiratory Biomedical Research Unit, Royal Brompton and Harefield NHS Foundation Trust and Imperial College, London, UK

${ }^{4}$ Guy's and St Thomas' NHS Foundation Trust and King's College London, National Institute of Health Research Comprehensive Biomedical Research Centre, London, UK

\section{Correspondence to} Dr Patrick B Murphy, Lane Fox Respiratory Unit, St Thomas' Hospital, Westminster Bridge Road, London SE1 7EH, UK; patrick.b.murphy@kcl.ac.uk

Received 8 September 2011 Accepted 26 January 2012 Published Online First

1 March 2012

\section{ABSTRACT}

Introduction Automatic titration modes of non-invasive ventilation, including average volume assured pressure support (AVAPS), are hybrid technologies that target a set volume by automated adjustment of pressure support (PS). These automated modes could offer potential advantages over fixed level PS, in particular, in patients who are super obese.

Methods Consecutive patients with obesity hypoventilation syndrome were enrolled in a two-centre prospective single-blind randomised controlled trial of AVAPS versus fixed-level PS using a strict protocolised setup.

Measurements The primary outcome was change in daytime arterial $\mathrm{PCO}_{2}\left(\mathrm{PaCO}_{2}\right)$ at 3 months. Body composition, physical activity (7-day actigraphy) and health-related quality of life (severe respiratory insufficiency questionnaire, SRI) were secondary outcome measures.

Results 50 patients (body mass index $50 \pm 7 \mathrm{~kg} / \mathrm{m}^{2}$ $55 \pm 11$ years; $53 \%$ men) were enrolled with a mean $\mathrm{PaCO}_{2}$ of $6.9 \pm 0.8 \mathrm{kPa}$ and $\mathrm{SRI}$ of $53 \pm 17.46$ patients (23 AVAPS and 23 PS) completed the trial. At 3 months, improvements in $\mathrm{PaCO}_{2}$ were observed in both groups (AVAPS $\triangle 0.6 \mathrm{kPa}, 95 \% \mathrm{Cl} 0.2$ to $1.1, \mathrm{p}<0.01$ vs PS $\Delta 0.6 \mathrm{kPa}, 95 \% \mathrm{Cl} 0.1$ to $1.1, \mathrm{p}=0.02)$ but no betweengroup difference $(\Delta-0.1 \mathrm{kPa}, 95 \% \mathrm{Cl}-0.7$ to 0.6 , $\mathrm{p}=0.87$ ). SRI also improved in both groups (AVAPS $\Delta 11$, $95 \% \mathrm{Cl} 6$ to $17, \mathrm{p}<0.001$ vs PS $\Delta 7,95 \% \mathrm{Cl} 1$ to 12 , $\mathrm{p}=0.02$; between groups $\Delta 5,95 \% \mathrm{Cl}-3$ to 12 , $p=0.21)$. Secondary analysis of both groups combined showed improvements in daytime physical activity that correlated with reduction in fat mass $(r=0.48 ; p=0.01)$.

Conclusion The study demonstrated no differences between automated AVAPS mode and fixed-level PS mode using a strict protocolised setup in patients who were super obese. The data suggest that the management of sleep-disordered breathing may enhance daytime activity and promote weight loss in super-obese patients. Trial registration details available at http:// www.controlled-trials.com/ISRCTN63940700

\section{INTRODUCTION}

Although only first described as a case report in $1955,{ }^{1}$ obesity hypoventilation syndrome (OHS)

\section{Key messages}

What is the key question?

- Does the addition of volume-targeted noninvasive ventilation (NIV) to standard fixed bi-level pressure support (PS) improve physiological and clinical outcomes in the treatment of stable obesity hypoventilation syndrome (OHS)?

What is the bottom line?

- There were no clinically important differences between volume-targeted NIV and fixed PS NIV when set up using a strict titration protocol in terms of improvement in gas exchange, daytime somnolence, health-related quality of life, and actigraphy assessed sleep and activity parameters.

\section{Why read on?}

- The study provides essential data for the clinician on the setup of NIV in OHS and confirms the improvements in gas exchange, daytime somnolence and health-related quality of life that occur with NIV therapy. It also demonstrates an objective improvement in daytime physical activity with the treatment of sleep-disordered breathing in $\mathrm{OHS}$.

has become the commonest diagnosis for initiation of domiciliary non-invasive ventilation (NIV). ${ }^{2}$ Data from physiological studies and controlled trials investigating the effect of continuous positive airways pressure (CPAP) and bi-level NIV in OHS have demonstrated improvements in daytime gas exchange and symptoms with both treatments. ${ }^{3-6}$ A single-centre randomised controlled trial of selected patients with OHS controlled on CPAP showed equivalence between CPAP and NIV, leading consensus opinion to advocate the use of NIV in patients whose condition is uncontrolled on CPAP. $^{7}$

Patients who are obese and have sleep-disordered breathing have a disturbance of the respiratory muscle load-capacity-drive relationship, resulting in chronic respiratory failure. ${ }^{8-10} \mathrm{We}$ 
previously demonstrated that in patients who are obese movement from sitting to supine increases respiratory muscle load and neural respiratory drive, with an associated fall in tidal volume, ${ }^{11}$ and this problem is further exaggerated through the different stages of sleep. ${ }^{12}$ Conceptually, standard bi-level NIV with fixed-level pressure support (PS) delivery may not maintain adequate ventilation during the changes in pulmonary mechanics that occur throughout sleep, so automatic titrating hybrid ventilatory modes that target a pre-set volume by adjustment of PS may be more effective. These novel modes, including average-volume-assured PS (AVAPS), estimate the expiratory tidal volume and respond by adjusting the inspiratory positive airway pressure (IPAP) to maintain ventilation. Although a small trial has shown that AVAPS provided a greater reduction in nocturnal transcutaneous carbon dioxide $\left(\mathrm{tcCO}_{2}\right)$ compared with fixed-level PS NIV in OHS, ${ }^{13}$ a subsequent study suggested that this improvement in ventilation was offset by greater sleep disruption as a consequence of the variation in PS delivered. ${ }^{14}$ This randomised controlled trial was undertaken to investigate if automated volume-targeted PS ventilation was more effective in reversing daytime hypercapnia than fixed-level PS ventilation using a strict protocolised setup in patients who are super obese and have chronic respiratory failure. Some of the results of this study have previously been reported in abstract form. ${ }^{15-17}$

\section{METHOD}

All subjects provided written informed consent prior to enrolment. The study was approved by Guy's Research Ethics Committee. The study was registered prospectively on a publically accessible database (http://www.controlled-trials.com/ ISRCTN63940700).

\section{Subjects}

Patients admitted to the Lane Fox Respiratory Unit, St Thomas' Hospital and to the Sleep and Ventilation Unit, Royal Brompton Hospital for either elective assessment of stable OHS or assessment following an episode of acute decompensated respiratory failure secondary to OHS were screened for study inclusion. Study inclusion criteria were body mass index $>40 \mathrm{~kg} / \mathrm{m}^{2}$; daytime stable respiratory failure with $\mathrm{PaCO}_{2}$ $>6 \mathrm{kPa}$ and $\mathrm{pH}>7.35$; absence of another identifiable cause of hypoventilation; ratio of forced expiratory volume in $1 \mathrm{~s}\left(\mathrm{FEV}_{1}\right)$ to forced vital capacity (FVC) $>0.70$; and FVC $<70 \%$ predicted. The exclusion criterion was an inability to provide written informed consent.

\section{Study design}

The study was a single (subject) blind, prospective, randomised controlled trial. Patients were randomly allocated to either fixed bi-level PS or AVAPS mode. Both modes were delivered by a BiPAP synchrony device (Philips-Respironics, Murrysville, Pennsylvania, USA). Pre-randomisation minimisation was performed to avoid allocation bias; variables were body mass index $\left(40-50 \mathrm{~kg} / \mathrm{m}^{2}, \quad 50-60 \mathrm{~kg} / \mathrm{m}^{2}\right.$ and $\left.>60 \mathrm{~kg} / \mathrm{m}^{2}\right)$, neck circumference $(<45 \mathrm{~cm}$ and $\geq 45 \mathrm{~cm})$, gender and clinical presentation (acute or elective).

\section{Patient assessment and treatment titration}

Patients underwent baseline assessments of spirometry (Microplus handheld spirometer, Cardinal Health, Dublin, Ohio, USA), arterial blood gas measurement and anthropometrics, including body composition measurements using the bioelectrical impedance method (Bodystat 1500, Bodystat Ltd, Isle of Man, UK). Health-related quality of life (HROL) was assessed by the severe respiratory insufficiency questionnaire (SRI). ${ }^{18}$ In addition, Epworth sleepiness score, fatigue severity score and visual analogue scales were recorded to assess selfreported sleep comfort, fatigue and physical activity levels. Following randomisation, patients underwent attended limited respiratory polygraphy, including oximetry and measurement of $\mathrm{tcCO}_{2}$ (Tosca 500, Radiometer, Crawley, UK) using an a priori protocol (figure $\mathrm{E} 1$, online data supplement) with settings titrated to abolish apnoeas and snoring and to achieve adequate nocturnal respiratory control. Supplementary oxygen was provided to patients who met the criteria for daytime hypoxaemia $\left(\mathrm{PaO}_{2}<7.3 \mathrm{kPa}\right.$ or $<8 \mathrm{kPa}$ with secondary features of hypoxia or right heart failure) at the lowest flow rate that corrected hypoxaemia $\left(\mathrm{PaO}_{2}>8 \mathrm{kPa}\right)$. Once established on NIV, patients were discharged and followed up at 3 months. A subset of patients underwent assessment of sleep disruption and daytime activity using the Actiwatch-64 (Philips-Respironics), an accelerometer device that has been used previously to measure daytime activity in obesity ${ }^{19}$ and to assess sleep patterns in respiratory sleep disorders. ${ }^{20}$ The accelerometer was worn for the 7 days following initiation of NIV and the 7 days following the 3-month assessment. Details of actigraphy analysis are provided in the online data supplement.

\section{Data analysis and statistics}

The study was powered with daytime ( $>4$ h post waking) $\mathrm{PaCO}_{2}$ as the primary outcome variable. Fifty patients were targeted for recruitment based on detecting a difference in daytime $\mathrm{PaCO}_{2}$ of $\geq 0.5 \mathrm{kPa}$ between the AVAPS and fixed-level PS groups. This provided a power of $80 \%$ at the $5 \%$ significance level and incorporated an expected $15 \%$ dropout rate. Data were analysed using independent or paired t test if appropriate, unless demonstrably not normally distributed, in which case an appropriate nonparametric equivalent was used. Parametric data are presented as mean \pm SD and non-parametric data as median (range). Correlation analyses were performed using Pearson's correlation test. For all analyses, a $\mathrm{p}$ value $<0.05$ was considered statistically significant. Data analyses were conducted using PASW statistics version 18 (SPSS, Chicago, IL, USA).

\section{RESULTS}

Sixty-two patients were screened for study participation. Fifty patients consented and underwent randomisation. Four patients (two from each group) withdrew during follow-up. Further details are provided in the CONSORT diagram (figure 1).

\section{Baseline anthropometrics and sleep variables}

The groups were matched for important variables at baseline (table 1).

\section{Automated versus protocolised NIV titration}

The median time to achieve satisfactory NIV setup was 2 days (range 1-4 days) for both groups. Nocturnal ventilatory control, assessed by overnight oximetry and $\mathrm{tcCO}_{2}$, was similar in both the AVAPS and fixed-level PS groups (table 2). Three patients failed to reach the predetermined criteria for satisfactory ventilator setup (2/25 PS vs $1 / 25$ AVAPS; $p=0.6)$ due to an inability to tolerate the increase in IPAP or estimated tidal volume (Vte). These patients were discharged on the highest tolerated settings. Supplementary oxygen was required by six patients (4/25 PS vs 2/25 AVAPS; $p=0.4$ ). 
Figure 1 Consort recruitment diagram for enrolment and follow-up. AVAPS, average-volume-assured pressure support; PS, pressure support.

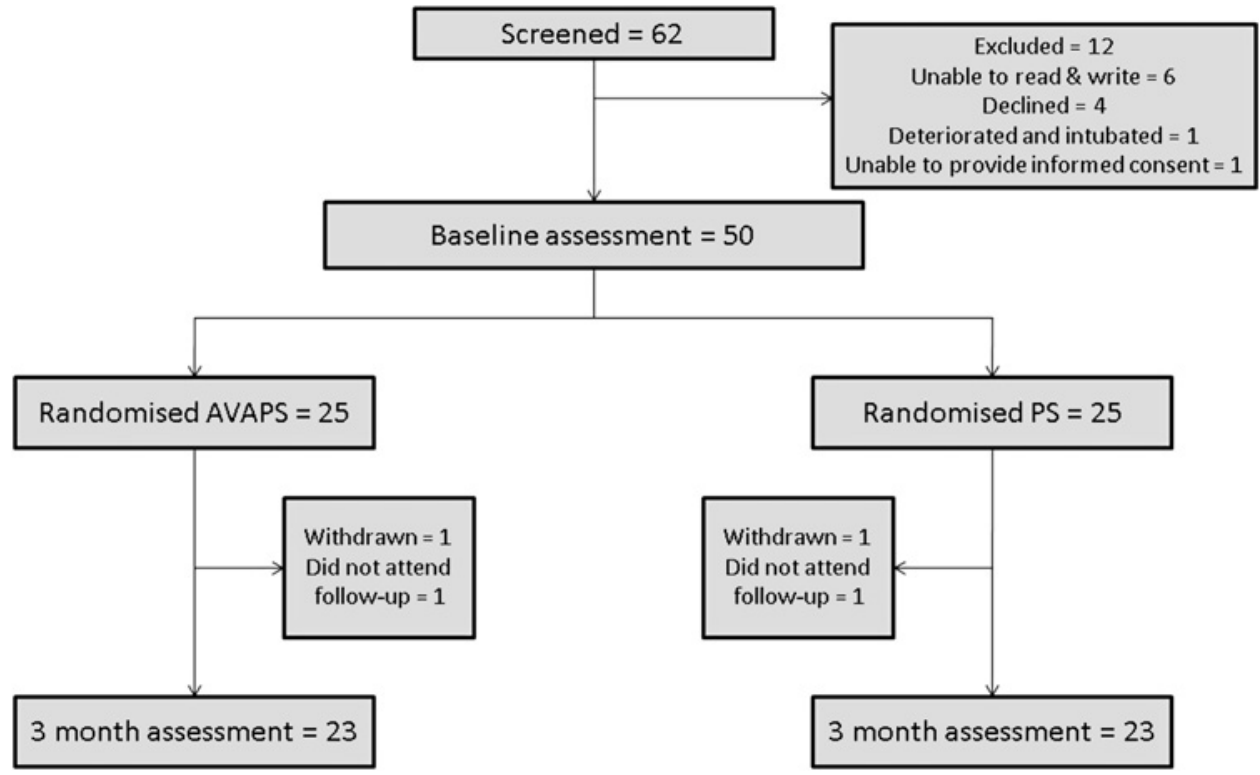

At discharge, ventilator settings provided a mean IPAP $25 \pm 3 \mathrm{cmH}_{2} \mathrm{O}$ in the fixed PS group and mean Vte of $657 \pm 96 \mathrm{ml}$ in the AVAPS group. A small difference in mean expiratory positive airway pressure was shown with $10 \pm 2 \mathrm{cmH}_{2} \mathrm{O}$ and $9 \pm 1 \mathrm{cmH}_{2} \mathrm{O}$ in the fixed-level PS and AVAPS groups, respectively $(p=0.03)$. Mean backup rate was $14 \pm 1$ breaths per minute in both groups.

\section{Clinical presentation}

The proportions of patients enrolled following an acute episode of decompensated hypercapnic respiratory failure were not significantly different between AVAPS and fixed PS groups $(p=0.77)$. Further details are available in the online data supplement.

\section{Actigraphy assessed sleep and physical activity parameters following initiation of NIV}

There were no significant differences demonstrated between AVAPS and fixed-level PS groups in estimated total sleep time, wake after sleep onset time, sleep efficiency and sleep latency, at baseline or follow-up (table 3).
Outcome following 3 months of domiciliary NIV

Gas exchange, HROL, daytime somnolence and control of sleepdisordered breathing

There were no-between group differences from baseline to follow-up in the primary outcome, $\mathrm{PaCO}_{2}$, or secondary outcomes: daytime gas exchange, anthropometric measures, spirometry, HROL or daytime somnolence (table 4 and table E1).

There were significant within-group improvements in $\mathrm{PaCO}_{2}$ (table 1), HROL (table 5), daytime somnolence, oximetry and $\mathrm{tcCO}_{2}$ (figure 2) between baseline and follow-up.

There were no between-group differences in any of the assessed outcome variables (table 1) or ventilator parameters (table 6).

Anthropometrics and physical activity

Thirty-two patients completed actigraphy monitoring analysis at baseline and 28 patients at follow-up. There were no differences between the AVAPS and fixed-level PS group at either baseline or follow-up in measures of daytime physical activity and anthropometric variables (table E5).

Table 1 Clinical variables at baseline and at 3-month follow-up

\begin{tabular}{|c|c|c|c|c|c|c|}
\hline & \multicolumn{3}{|l|}{ AVAPS } & \multicolumn{3}{|c|}{ Fixed-level PS } \\
\hline & Baseline & Follow-up & $\overline{p \text { Value }}$ & Baseline & Follow-up & p Value \\
\hline Age (years) & $53 \pm 9$ & & & $56 \pm 11$ & & \\
\hline Gender (male/female) & $12 / 13$ & & & $11 / 14$ & & \\
\hline Emergency/elective presentation & $9 / 16$ & & & $9 / 16$ & & \\
\hline BMI $\left(\mathrm{kg} / \mathrm{m}^{2}\right)$ & $50 \pm 8$ & $48 \pm 9$ & 0.007 & $52 \pm 8$ & $51 \pm 7$ & 0.024 \\
\hline Fat-free mass $(\mathrm{kg})$ & $69 \pm 17$ & $68 \pm 18$ & 0.493 & $72 \pm 18$ & $71 \pm 18$ & 0.870 \\
\hline Waist circumference $(\mathrm{cm})$ & $141 \pm 18$ & $136 \pm 17$ & 0.006 & $145 \pm 14$ & $146 \pm 14$ & 0.120 \\
\hline Neck circumference $(\mathrm{cm})$ & $46 \pm 6$ & $46 \pm 7$ & 0.340 & $48 \pm 5$ & $48 \pm 7$ & 0.084 \\
\hline $\mathrm{FEV}_{1}(\%$ predicted $)$ & $53 \pm 15$ & $59 \pm 14$ & 0.039 & $55 \pm 15$ & $60 \pm 16$ & 0.222 \\
\hline FVC (\% predicted) & $52 \pm 14$ & $58 \pm 13$ & 0.019 & $56 \pm 15$ & $62 \pm 18$ & 0.189 \\
\hline $\mathrm{PaCO}_{2}(\mathrm{kPa})$ & $7.0 \pm 0.7$ & $6.4 \pm 0.8$ & 0.004 & $6.8 \pm 0.8$ & $6.2 \pm 0.8$ & 0.021 \\
\hline $\mathrm{PaO}_{2}(\mathrm{kPa})$ & $8.9 \pm 1.2$ & $9.1 \pm 1.2$ & 0.660 & $8.7 \pm 1.8$ & $9.3 \pm 1.2$ & 0.163 \\
\hline $\mathrm{HCO}_{3}$ (mmol/litre) & $31 \pm 3$ & $29 \pm 3$ & 0.001 & $31 \pm 4$ & $27 \pm 3$ & 0.003 \\
\hline
\end{tabular}

The $p$ values refer to paired $t$ test analysis from initiation to follow-up values within each group.

AVAPS, average-volume-assured pressure support: $\mathrm{BMl}$, body mass index; FEV ${ }_{1}$, forced expiratory volume in $1 \mathrm{~s}$; FVC, forced vita capacity; $\mathrm{HCO}_{3}$, arterial concentration of bicarbonate; $\mathrm{PaCO}_{2}$, arterial partial pressure of carbon dioxide; $\mathrm{PaO}_{2}$, arterial partial pressure of oxygen; PS, pressure support. 
Table 2 Comparison of oximetry-capnometry measurements prior to discharge following automated AVAPS setup and protocolised fixed-level PS setup

\begin{tabular}{|c|c|c|c|}
\hline & AVAPS & Fixed-level PS & p Value \\
\hline $4 \%$ ODI (events/h) & $22 \pm 16$ & $22 \pm 17$ & 0.517 \\
\hline Mean $\mathrm{SpO}_{2}(\%)$ & $92 \pm 3$ & $92 \pm 3$ & 0.552 \\
\hline$\%$ TST $<90 \%(\%)$ & $20 \pm 21$ & $15 \pm 20$ & 0.630 \\
\hline Mean tcCO ${ }_{2}(\mathrm{kPa})$ & $7.1 \pm 0.7$ & $7.2 \pm 1$ & 0.952 \\
\hline $\operatorname{Max~tcCO}{ }_{2}(\mathrm{kPa})$ & $8.4 \pm 0.8$ & $8.4 \pm 1.6$ & 0.980 \\
\hline
\end{tabular}

The $\mathrm{p}$ value refers to comparison between interventions by independent $t$ test. AVAPS, average-volume-assured pressure support; \% $0 \mathrm{DI}$, 4\% oxygen desaturation index; PS, pressure support; $\mathrm{SpO}_{2}$, oxygen saturation of haemoglobin; $\mathrm{tcCO}_{2}$, transcutaneous carbon dioxide; $\%$ TST $<90 \%$, \% total sleep time with $\mathrm{SpO}_{2}$ under $90 \%$.

\section{Combined AVAPS and fixed-level PS cohort}

As there were no clinically significant differences demonstrated between the AVAPS and fixed-level PS modes in either primary or secondary outcomes, a single cohort $(n=28)$ was produced to allow a post hoc analysis of the relationship between physical activity and NIV in patients with OHS.

\section{Physical activity and weight loss}

Baseline data showed that patients spent an average of $3 \mathrm{~h}$ $21 \mathrm{~min} \pm 1 \mathrm{~h} 33 \mathrm{~min}$ immobile or asleep during the daytime period. There were significant inverse correlations observed between daytime activity (mean activity counts per day), and weight $(\mathrm{r}=-0.39 ; \mathrm{p}=0.02)$ and waist circumference $(\mathrm{r}=-0.42$; $\mathrm{p}=0.01$ ). Significant reductions in weight, fat mass and waist circumference were observed following 3 months of NIV, with an associated increase in physical activity (table 7).

There were correlations between change in physical activity, as measured by change in immobile time, and both the change in fat mass $(r=0.48 ; p=0.01)$ and waist circumference $(r=0.46$; $\mathrm{p}=0.01$ ) between baseline and follow-up assessment (figure 3).

\section{Dose response to NIV}

NIV showed a dose response effect with a significant correlation between hours of use and improvement in daytime $\mathrm{PaCO}_{2}$ $(r=-0.37 ; p=0.01)$ with the $95 \%$ CI crossing below 0 with an adherence time of $4 \mathrm{~h}$ (figure E2). Calculated mean Vte per $\mathrm{kg}$ ideal body weight (IBW) during the 3-month trial period correlated with change in daytime $\mathrm{PaCO}_{2}(\mathrm{r}=0.39 ; \mathrm{p}=0.01)$ from baseline to follow-up (figure E3). These data indicate that $10 \mathrm{ml} /$ $\mathrm{kg} I B W$ is the most appropriate ventilator setting in the AVAPS mode.

\section{Backup rate and controlled nocturnal ventilation}

Both the AVAPS and fixed-level PS groups had similar pre-set backup rates with similar levels of patient-triggered breaths. A post hoc analysis of the combined AVAPS and fixed-level PS
Table 4 Changes in gas exchange, anthropometrics, spirometry, daytime somnolence and HROL between NIV initiation and follow-up

\begin{tabular}{lccll}
\hline & & $\begin{array}{l}\text { Fixed-level } \\
\text { PS }\end{array}$ & $\begin{array}{l}\text { Mean difference } \\
\text { between treatments } \\
\text { (95\% Cl) }\end{array}$ & p Value \\
\hline$\Delta \mathrm{PaCO}_{2}(\mathrm{kPa})$ & $-0.6 \pm 1.0$ & $-0.6 \pm 1.1$ & $0(-0.7$ to 0.6$)$ & 0.867 \\
$\Delta \mathrm{PaO}_{2}(\mathrm{kPa})$ & $0.2 \pm 1.7$ & $0.5 \pm 1.6$ & $0(-1$ to 1$)$ & 0.519 \\
$\Delta \mathrm{HCO}_{3}$ (mmol/litre) & $-3 \pm 3$ & $-3 \pm 4$ & $0(-2$ to 2$)$ & 0.825 \\
$\Delta \mathrm{BMI}\left(\mathrm{kg} / \mathrm{m}^{2}\right)$ & $-1 \pm 2$ & $-2 \pm 4$ & $1(-1$ to 2$)$ & 0.497 \\
$\Delta$ Fat free mass (kg) & $-1 \pm 6$ & $0 \pm 8$ & $-1(-4$ to 3$)$ & 0.805 \\
$\Delta$ Waist & $-3 \pm 5$ & $-2 \pm 7$ & $-1(-3$ to 4$)$ & 0.676 \\
circumference (cm) & & & & \\
$\Delta \mathrm{FEV}{ }_{1}(\%$ predicted) & $6 \pm 13$ & $4 \pm 14$ & $2(-6$ to 10$)$ & 0.588 \\
$\Delta \mathrm{FVC} \mathrm{( \%} \mathrm{predicted)}$ & $6 \pm 12$ & $5 \pm 17$ & $1(-7$ to 10$)$ & 0.777 \\
$\Delta \mathrm{ESS}(/ 24)$ & $-5 \pm 6$ & $-6 \pm 6$ & $1(-2$ to 5$)$ & 0.428 \\
$\Delta \mathrm{SRI}-\mathrm{SS}(/ 100)$ & $11 \pm 12$ & $7 \pm 13$ & $5(-2$ to 12$)$ & 0.212 \\
\hline
\end{tabular}

The $\mathrm{p}$ value refers to comparison between interventions by independent $t$ test. AVAPS, average-volume-assured pressure support; BMI, body mass index; ESS, Epworth Sleepiness Score (higher values indicate higher degrees of daytime somnolence); FEV forced expiratory volume in $1 \mathrm{~s}$; $\mathrm{FVC}$, forced vital capacity; $\mathrm{HCO}_{3}$, arterial concentration of bicarbonate; $\mathrm{HROL}$, health-related quality of life; $\mathrm{PaCO}_{2}$, arterial partial pressure of carbon dioxide; $\mathrm{PaO}_{2}$, arterial partial pressure of oxygen; PS, pressure support; SRI-SS, Severe Respiratory Insufficiency questionnaire summary score (higher values indicate better healthrelated quality of life).

cohort investigating the frequency of dependence on backup rate pressure controlled ventilation is provided in the online data supplement (tables E6-E8). Patients with a backup rate pressure controlled ventilation dependency $>50 \%$ had a greater control of nocturnal carbon dioxide, improved daytime carbon dioxide and enhanced HROL at 3 months.

\section{DISCUSSION}

This single-blind randomised controlled trial demonstrated that AVAPS ventilation has similar efficacy to fixed-level PS ventilation when accompanied by a strict protocolised setup in reducing daytime carbon dioxide level in patients with super obesity and OHS. These results are in contrast to previous data suggesting that automated variable PS provided enhanced nocturnal ventilatory control, but at a cost of increased sleep disruption. The current data represent the largest randomised controlled trial in patients with super obesity and chronic respiratory failure, and as such, these clinical data significantly add to the limited published data available. In addition, these data confirm the findings of previous small studies in patients who are less obese, demonstrating the improvements in daytime gas exchange, daytime somnolence and HROL that can be achieved with the use of bi-level NIV. Furthermore in contrast to previous data, this study indicates that nocturnal treatment of chronic respiratory failure in patients who are super obese enhances daytime physical activity, which is associated with weight loss.

Table 3 Actigraphy analysed sleep parameters for the first week following initiation of NIV compared with the first week following the 3-month assessment in the AVAPS $(n=14)$ and fixed-level PS $(n=15)$ arms

\begin{tabular}{|c|c|c|c|c|c|c|}
\hline & \multicolumn{2}{|l|}{ Baseline } & \multirow[b]{2}{*}{ p Value } & \multicolumn{2}{|c|}{ Follow-up } & \multirow[b]{2}{*}{ p Value } \\
\hline & AVAPS & Fixed-level PS & & AVAPS & Fixed-level PS & \\
\hline TST (min) & $341 \pm 80$ & $352 \pm 78$ & 0.713 & $321 \pm 52$ & $346 \pm 75$ & 0.302 \\
\hline WASO\%TST (\%) & $23 \pm 11$ & $23 \pm 17$ & 0.987 & $27 \pm 16$ & $20 \pm 13$ & 0.185 \\
\hline Latency (min) & $5 \pm 3$ & $8 \pm 7$ & 0.164 & $4 \pm 2$ & $5 \pm 6$ & 0.577 \\
\hline Efficiency (\%) & $80 \pm 7$ & $80 \pm 13$ & 0.894 & $79 \pm 9$ & $81 \pm 9$ & 0.416 \\
\hline
\end{tabular}

The $p$ value refers to comparison between interventions at each time point by independent $t$ test.

AVAPS, average-volume-assured pressure support; PS, pressure support; TST, total sleep time; WASO\%TST, wake after sleep onset as a \% of TST. 
Table 5 HRQL pre-post treatment in AVAPS and PS groups

\begin{tabular}{|c|c|c|c|c|c|c|}
\hline & \multicolumn{3}{|l|}{ AVAPS } & \multicolumn{3}{|c|}{ Fixed-level PS } \\
\hline & Baseline & Follow-up & p Value & Baseline & Follow-up & p Value \\
\hline SRI-SS (/100) & $55 \pm 16$ & $66 \pm 19$ & $<0.001$ & $51 \pm 14$ & $57 \pm 15$ & 0.018 \\
\hline SRI-RC (/100) & $55 \pm 20$ & $70 \pm 20$ & 0.001 & $49 \pm 24$ & $59 \pm 22$ & 0.025 \\
\hline SRI-PF (/100) & $50 \pm 24$ & $58 \pm 26$ & 0.069 & $42 \pm 20$ & $47 \pm 22$ & 0.139 \\
\hline SRI-AS (/100) & $48 \pm 17$ & $62 \pm 20$ & 0.003 & $48 \pm 19$ & $54 \pm 16$ & 0.100 \\
\hline SRI-SR (/100) & $66 \pm 20$ & $72 \pm 24$ & 0.116 & $67 \pm 20$ & $73 \pm 18$ & 0.165 \\
\hline SRI-AX (/100) & $48 \pm 24$ & $65 \pm 29$ & 0.001 & $41 \pm 23$ & $50 \pm 21$ & 0.094 \\
\hline SRI-SF (/100) & $61 \pm 24$ & $73 \pm 20$ & 0.005 & $55 \pm 22$ & $63 \pm 22$ & 0.143 \\
\hline VAS-sleep comfort (/100) & $44 \pm 30$ & $57 \pm 27$ & 0.026 & $33 \pm 27$ & $53 \pm 22$ & 0.001 \\
\hline VAS-activity (/100) & $43 \pm 24$ & $52 \pm 26$ & 0.177 & $47 \pm 23$ & $47 \pm 22$ & 0.967 \\
\hline VAS-fatigue (/100) & $39 \pm 23$ & $59 \pm 27$ & 0.001 & $42 \pm 26$ & $55 \pm 28$ & 0.058 \\
\hline ESS $(/ 24)$ & $11 \pm 5$ & $6 \pm 5$ & 0.001 & $13 \pm 6$ & $7 \pm 5$ & $<0.001$ \\
\hline FSS $(/ 56)$ & $43 \pm 14$ & $34 \pm 15$ & 0.014 & $45 \pm 16$ & $37 \pm 18$ & 0.038 \\
\hline
\end{tabular}

The $\mathrm{p}$ values refer to paired $\mathrm{t}$ test analysis from initiation to follow-up values within each group.

AVAPS, average-volume-assured pressure support; ESS, Epworth Sleepiness Score; FSS, fatigue severity score (total=56; higher score indicates greater level of fatigue); HROL, health-related quality of life; PS, pressure support; SRI-AS, Severe Respiratory Insufficiency questionnaire attendant symptoms and sleep; SRI-AX, Severe Respiratory Insufficiency questionnaire anxiety; SRI-PF, Severe Respiratory Insufficiency questionnaire physical functioning; SRI-RC, Severe Respiratory Insufficiency questionnaire respiratory complaints; SRI-SR, Severe Respiratory Insufficiency questionnaire social relationships; SRI-SS, Severe Respiratory Insufficiency questionnaire summary scale (total=100; higher score indicates higher quality of life); SRI-WB, Severe Respiratory Insufficiency questionnaire psychological wellbeing; SRI-SF, Severe Respiratory Insufficiency questionnaire social functioning; VAS, visual analogue scale (higher score indicates greater quality of life).

\section{Critique of method}

\section{Study design}

The study design used was a single-blind randomised controlled trial. The primary outcome was objective and all other assess- ments were conducted in accordance with international guidelines, when available, or local policies to minimise the chance of assessor bias. This limitation is constant throughout other randomised studies in this area. ${ }^{3} 13$ Although the scientific
Figure 2 Pre-post treatment effect of pressure support (PS) and averagevolume-assured pressure support (AVAPS) modes on data collected from combined oximetry-capnometry during ventilation: (A) nocturnal mean oxygen saturation $\left(\mathrm{SpO}_{2}\right)$ during ventilation; $(\mathrm{B})$ $\%$ sleep time with $\mathrm{SpO}_{2}<90 \%$ during ventilation; (C) mean transcutaneous carbon dioxide $\left(\mathrm{tcCO}_{2}\right)$ during ventilation; (D) maximum transcutaneous carbon dioxide $\left(\mathrm{tcCO}_{2}\right)$ during ventilation.
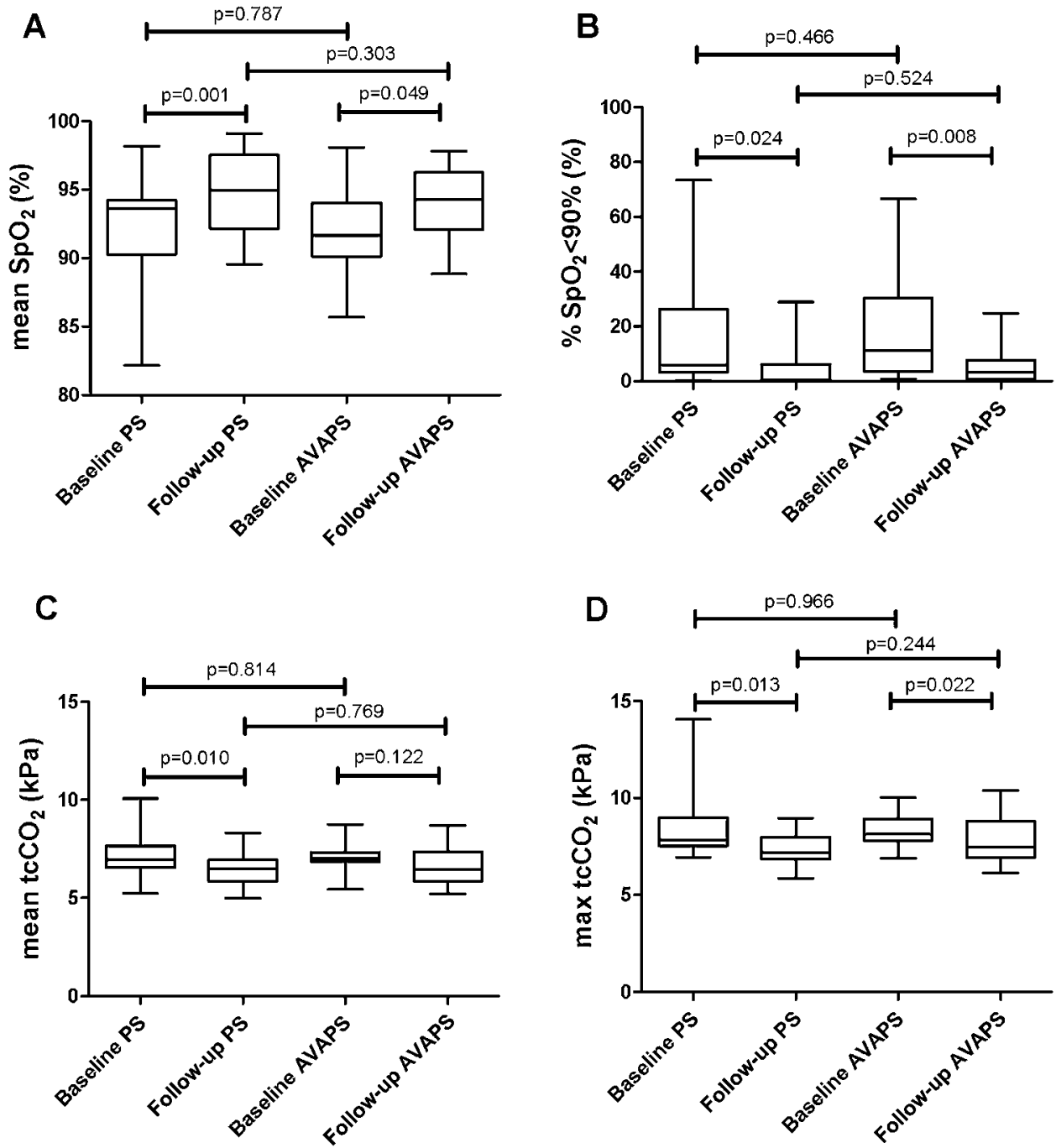
Table 6 Ventilator parameters at follow-up

\begin{tabular}{llll}
\hline & AVAPS & Fixed-level PS & p Value \\
\hline Delivered IPAP $\left(\mathrm{cmH}_{2} \mathrm{O}\right)$ & $22 \pm 5$ & $23 \pm 4$ & 0.402 \\
Leak (litres/min) & $53 \pm 13$ & $53 \pm 19$ & 0.968 \\
Patient-triggered breaths $(\%)$ & $43 \pm 27$ & $45 \pm 27$ & 0.759 \\
Compliance (h:min/day) & $4: 11 \pm 02: 53$ & $5: 08 \pm 02: 22$ & 0.230 \\
\hline
\end{tabular}

AVAPS, average-volume-assured pressure support; IPAP, inspiratory positive airway pressure; PS, pressure support.

quality of the current trial would have been enhanced by the addition of a third control arm, the clinical consensus and current evidence strongly support the use of domiciliary NIV in patients with significant nocturnal hypoxia and hypercapnia, such as were enrolled in this study. Furthermore, survival data from observational studies have demonstrated that in this patient population NIV confers a survival advantage and therefore we considered that the use of a control arm raised considerable clinical safety and ethical concerns. ${ }^{21} 22$ This is the largest randomised controlled trial in this area and although it was designed with an $80 \%$ power there was a lower than expected dropout rate. However, the failure to demonstrate treatment superiority of AVAPS could occur as a result of a type 2 error

\section{Assessment methods}

For the purposes of this study, we were focused on the primary outcome of change in $\mathrm{PaCO}_{2}$ and differences in nocturnal ventilatory control between the AVAPS and the fixed-level PS, assessed and titrated using limited attended respiratory polygraphy. While it is considered ideal to confirm sleep and quantify sleep staging using extended polysomnography, this should not detract from the findings of the current trial. Previous studies have shown that oximetry-capnometry is an accurate method to monitor change in $\mathrm{tcCO}_{2}$ in patients who are obese during NIV initiation. ${ }^{23-26}$ The limitations of extended polysomnography to assess ventilator-induced sleep disturbance must also be highlighted. This approach only provides a single night assessment in a monitored hospital setting with a substantial array of electrocephalic, electromyographic and respiratory physiological monitoring equipment attached to the patient, all of which may cause sleep disruption. Despite the difficulties of assessing the disruptive effects of NIV on sleep, we aimed to investigate the effect of AVAPS and fixed-level PS on nocturnal disruption using 7-day actigraphy. As this technique collects data over multiple nights in the home, overcoming the nightly variation occurring with polysomnography, ${ }^{27} 28$ it has been suggested to be a superior method of assessing treatmentassociated sleep disruption in OSA. ${ }^{29}$ Furthermore, actigraphy has been shown to be a valid method of assessing the sleepwake cycle in patients with sleep-disordered breathing 30 31
Table 7 Actigraphy $(n=28)$ and anthropometric variables $(n=46)$ at baseline and 3-month follow-up

\begin{tabular}{lccl}
\hline & Baseline & $\begin{array}{c}\text { Follow-up at } \\
\text { 3 months }\end{array}$ & p Value \\
\hline Weight (kg) & $141 \pm 28$ & $137 \pm 28$ & 0.001 \\
Fat-free mass (kg) & $70 \pm 17$ & $69 \pm 17$ & 0.593 \\
Fat mass (kg) & $70 \pm 21$ & $67 \pm 19$ & 0.041 \\
Waist circumference (cm) & $142 \pm 15$ & $140 \pm 16$ & 0.003 \\
Mean activity counts (counts/day) & $232 \pm 100$ & $263 \pm 94$ & 0.016 \\
Max activity counts (counts/day) & $1797 \pm 507$ & $2100 \pm 553$ & 0.006 \\
Immobile time (min/day) & $201 \pm 93$ & $161 \pm 84$ & 0.028 \\
Mobile time (min/day) & $771 \pm 86$ & $785 \pm 110$ & 0.417
\end{tabular}

Actigraphy analysed for the first week at home following initiation of non-invasive ventilation (NIV) compared with the first week following the 3-month assessment of NIV.

and the previously reported values for total sleep time, wake after sleep onset and sleep efficiency in patients with OHS using extended polysomnography are comparable with our current data. ${ }^{13} 14$ Actigraphy has the added benefit of providing objective daytime physical activity data and in the current study it permitted interrogation of the relationships between nocturnal ventilatory control, daytime somnolence, physical activity and weight loss.

\section{Applicability of findings}

This study was designed to investigate the effects of a specific, propriety ventilator technology compared with a standard protocolised ventilator setup in OHS. There are, however, other devices incorporating pressure-volume hybrid ventilatory modes. Although working through the same fundamental principles, each of these differ in the exact method used to estimate and correct tidal volume. Therefore, the results of this trial are only applicable to the AVAPS mode and cannot necessarily be extrapolated to all such devices.

\section{Significance of findings}

\section{Efficacy of ventilation}

Both NIV modes provided similar control of nocturnal ventilation at baseline and follow-up. This was reflected as a similar improvement in hypercapnia at 3 months. These data are in contrast to previous studies demonstrating greater reduction in transcutaneous carbon dioxide during volume-targeted PS ventilation compared with fixed-level PS ventilation. ${ }^{13}{ }^{14}$ However, neither of the previous trials used a study titration protocol, as was used in the current study, to minimise the differences between the groups, and thus the ventilator setup favoured higher levels of PS delivered in the volume-targeted PS ventilation arm resulting in greater carbon dioxide clearance. In addition to previously published data, the data from this study showed that $\geq 4$ h nocturnal ventilation was required to achieve
Figure 3 Correlations between change in daily physical activity as measured by daytime period spent immobile and $(A)$ change in fat mass: $r=0.48, p=0.01$; $(B)$ change in waist circumference: $r=0.46, p=0.01$.
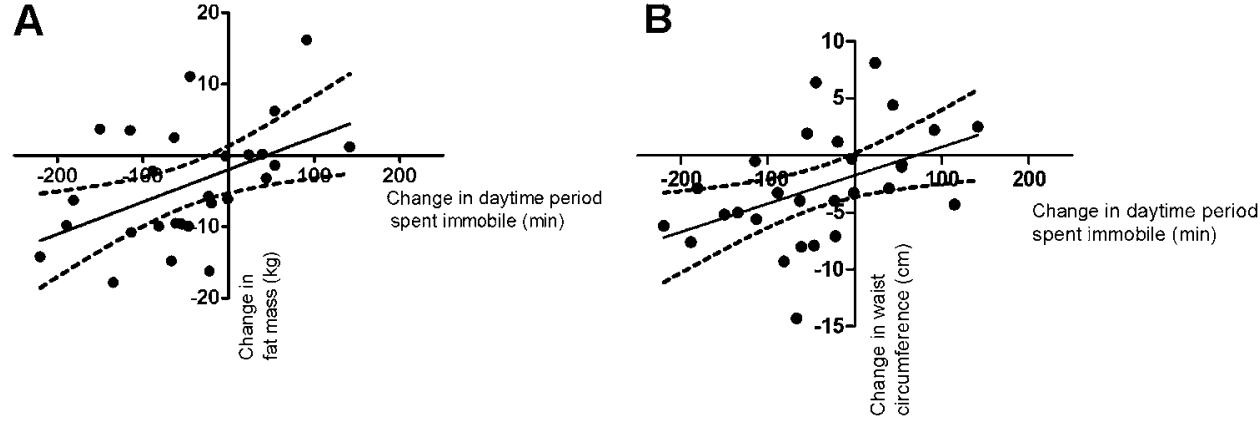
a reduction in daytime carbon dioxide. These data are highly relevant to clinicians managing patients who are super obese to satisfactorily prescribe bi-level NIV.

\section{Ventilation-induced nocturnal disruption}

This study challenges previous data that showed volumetargeted PS ventilation contributes to sleep disruption during initiation of NIV in patients with OHS. ${ }^{14}$ However, we consider our study design may explain this discrepancy because the previous studies used a crossover design, which is methodologically inferior to $1: 1$ randomisation. The patients were randomised in a crossover design to their 'normal' NIV with or without volume-targeted PS. Furthermore, the estimated tidal volume was based on the patients' actual body weight $(8-10 \mathrm{ml} / \mathrm{kg})$ rather than their IBW, which was the method used in the current study. Not unexpectedly, this study design resulted in a significantly higher mean IPAP in the volumetargeted PS group. Therefore, in addition to the unfamiliar mode of ventilation, these patients were provided with ventilator settings likely to negatively impact on sleep quality. In contrast, the current study had an a priori protocol for titration to produce similar delivered mean PS levels between the groups. Therefore, the data suggest that the differences demonstrated in earlier studies were inherent to study design and setup protocol rather than the treatment mode per se.

Improvements in HRQL, daytime somnolence and physical activity Consistent with previous reports, there was a significant treatment effect resulting in improvements in daytime somnolence and HRQL. ${ }^{3} 13$ There were no differences demonstrated in the magnitude of these improvements between groups, as expected, given that the efficacy of ventilation was similar. Our data suggest that nocturnal ventilatory support improves daytime symptoms and physical activity and that this is associated with weight loss in people who are obese. Interestingly, the observed change in body composition was a change in fat mass, rather than fat-free mass, indicating that it is not the result of the extracellular fluid clearance that would be expected to accompany the resolution of right ventricular dysfunction and cor pulmonale. The increased physical activity and reduction in weight accompanying the use of NIV for $\geq 4 \mathrm{~h}$ per night in patients who are super obese with OHS could potentially be enhanced by cardiopulmonary rehabilitation, and this needs to be considered in future studies. ${ }^{32}$

\section{Conclusion}

This single-blind randomised controlled trial showed that automated volume-targeted PS ventilation is as effective as standard fixed bi-level PS ventilation accompanied by a strict protocolised setup, producing similar improvements in nocturnal ventilatory control and daytime gas exchange, HROL, daytime symptoms and daytime physical activity. In addition, the level of nocturnal disruption with both modes was similar. As a cohort sample, these data inform clinical practice by defining the duration of nocturnal NIV adherence to reduce daytime arterial carbon dioxide and improve daytime symptoms. Finally, this study has shown that improved control of sleep-disordered breathing in OHS was associated with an increase in physical activity and subsequent weight loss, highlighting the potential for augmenting this effect by the addition of an exercise programme.

Contributors Conception and design: NH, MIP, JM; data collection: PM; analysis and data interpretation: PM, NH, MIP, JM; manuscript drafting: PM, ACD, AS, MH, NSH, AJW, JM, MIP, NH.
Funding The study was supported by an unrestricted educational grant from Philips-Respironics. The funder was not involved in study design, data collection or analysis, decision to publish or preparation of this manuscript. Philips-Respironics provided the BiPAP Synchrony and Actiwatch 64 devices used in the study. The authors acknowledge financial support from the Department of Health via the National Institute for Health Research (NIHR) comprehensive Biomedical Research Centre award to Guy's and St Thomas' NHS Foundation Trust in partnership with King's College London and King's College Hospital NHS Foundation Trust and the NIHR Respiratory Disease Biomedical Research Unit at the Royal Brompton and Harefield NHS Foundation Trust and Imperial College, London.

Competing interests The Lane Fox Translational Respiratory Research Group has received unrestricted research grants from ResMed, Abingdon, Oxfordshire, UK Philips-Respironics, Murrysville, Pennsylvania, USA; Fisher \& Paykel Healthcare, Auckland, New Zealand; and B \& D ElectroMedical, Stratford-upon-Avon,

Warwickshire, UK. PM has received expenses for travel to conferences from Philips-Respironics. AJW has received expenses for travel to conferences from ResMed. NH has received fees for lecturing from Philips-Respironics and Fisher \& Paykel. MIP has received fees for lecturing from Philips-Respironics. This study was supported by Philips-Respironics, Murrysville, Pennsylvania, USA. The study design, results, interpretation of the findings or any other subject discussed in the submitted manuscript were not dependent on support.

Ethics approval Guy's REC.

Provenance and peer review Not commissioned; externally peer reviewed.

\section{REFERENCES}

1. Auchincloss JH Jr, Cook E, Renzetti AD. Clinical and physiological aspects of a case of obesity, polycythemia and alveolar hypoventilation. J Clin Invest 1955; 34:1537-45

2. Janssens JP, Derivaz $S$, Breitenstein $E$, et al. Changing patterns in long-term noninvasive ventilation: a 7-year prospective study in the Geneva Lake area. Chest 2003;123:67-79

3. Piper AJ, Wang D, Yee BJ, et al. Randomised trial of CPAP vs bilevel support in the treatment of obesity hypoventilation syndrome without severe nocturnal desaturation. Thorax 2008;63:395-401.

4. Berger KI, Ayappa I, Chatr-Amontri B, et al. Obesity hypoventilation syndrome as a spectrum of respiratory disturbances during sleep. Chest 2001;120: 1231-8.

5. Sullivan CE, Berthon-Jones M, Issa FG. Remission of severe obesity-hypoventilation syndrome after short-term treatment during sleep with nasal continuous positive airway pressure. Am Rev Respir Dis 1983;128:177-81.

6. de Lucas-Ramos P, de Miguel-Diez J, Santacruz-Siminiani A, et al. Benefits at 1 year of nocturnal intermittent positive pressure ventilation in patients with obesityhypoventilation syndrome. Respir Med 2004;98:961-7.

7. Anon. Clinical indications for noninvasive positive pressure ventilation in chronic respiratory failure due to restrictive lung disease, COPD, and nocturnal hypoventilation-a consensus conference report. Chest 1999;116:521-34.

8. Sharp JT, Henry JP, Sweany SK, et al. The total work of breathing in normal and obese men. J Clin Invest 1964;43:728-39.

9. Leech J, Onal E, Aronson R, et al. Voluntary hyperventilation in obesity hypoventilation. Chest 1991;100:1334-8.

10. Sampson MG, Grassino K. Neuromechanical properties in obese patients during carbon dioxide rebreathing. Am J Med 1983;75:81-90.

11. Steier J, Jolley CJ, Seymour J, et al. Neural respiratory drive in obesity. Thorax 2009:64:719-25.

12. Resta 0, Foschino-Barbaro MP, Bonfitto $P$, et al. Prevalence and mechanisms of diurnal hypercapnia in a sample of morbidly obese subjects with obstructive sleep apnoea. Respir Med 2000;94:240-6.

13. Storre $\mathbf{J H}$, Seuthe B, Fiechter R, et al. Average volume-assured pressure support in obesity hypoventilation: a randomized crossover trial. Chest 2006;130:815-21.

14. Janssens JP, Metzger M, Sforza E. Impact of volume targeting on efficacy of bilevel non-invasive ventilation and sleep in obesity-hypoventilation. Respir Med 2009;103:165-72.

15. Murphy PB, Williams AJ, Davidson AC, et al. Investigating patient dependence on back up rate pressure controlled ventilation (PCV) in obesity hypoventilation syndrome (OHS). Eur Respir J 2010;36:655s.

16. Murphy PB, Davidson AC, Williams AJ, et al. Interim data from a randomised controlled trial of average volume-assured pressure support (AVAPS) versus spontaneous-timed (ST) pressure support in obesity hypoventilation syndrome (OHS) Thorax 2010;65:A31.

17. Murphy PB, Brignall K, Hind M, et al. Activity levels at the initiation of home mechanical ventilation in patients with obesity hypoventilation syndrome. Thorax 2009;64:A29.

18. Gosh D, Rzehak P, Elliott MW, et al. Validation of the English Severe Respiratory Insufficiency Questionnaire. Eur Respir J. Published Online First: 19 December 2011. doi:10.1183/009031936.00152411

19. Chen KY, Acra SA, Majchrzak K, et al. Predicting energy expenditure of physical activity using hip- and wrist-worn accelerometers. Diabetes Technol Ther 2003;5:1023-33 
20. Chae KY, Kripke DF, Poceta JS, et al. Evaluation of immobility time for sleep latency in actigraphy. Sleep Med 2009;10:621-5.

21. Nowbar S, Burkart KM, Gonzales R, et al. Obesity-associated hypoventilation in hospitalized patients: prevalence, effects, and outcome. Am J Med Genet 2004;116:1-7.

22. Budweiser S, Riedl SG, Jorres RA, et al. Mortality and prognostic factors in patients with obesity-hypoventilation syndrome undergoing noninvasive ventilation. $J$ Intern Med 2007;261:375-83.

23. Maniscalco $\mathbf{M}$, Zedda A, Faraone $\mathbf{S}$, et al. Evaluation of a transcutaneous carbon dioxide monitor in severe obesity. Intensive Care Med 2008;34:1340-4.

24. Senn 0, Clarenbach CF, Kaplan V, et al. Monitoring carbon dioxide tension and arterial oxygen saturation by a single earlobe sensor in patients with critical illness or sleep apnea. Chest 2005;128:1291-6.

25. Storre JH, Steurer B, Kabitz HJ, et al. Transcutaneous PCO2 monitoring during initiation of noninvasive ventilation. Chest 2007:132:1820-6.
26. Storre $\mathbf{J H}$, Magnet FS, Dreher $\mathbf{M}$, et al. Transcutaneous monitoring as a replacement for arterial PCO(2) monitoring during nocturnal non-invasive ventilation. Respir Med 2011;105:143-50.

27. Aber WR, Block AJ, Hellard DW, et al. Consistency of respiratory measurements from night to night during the sleep of elderly men. Chest 1989;96:747-51.

28. Bittencourt LR, Suchecki D, Tufik S, et al. The variability of the apnoea-hypopnoea index. J Sleep Res 2001;10:245-51.

29. Kline CE, Crowley EP, Ewing GB, et al. The effect of exercise training on obstructive sleep apnea and sleep quality: a randomized controlled trial. Sleep 2011:34:1631-40.

30. Tryon WW. Issues of validity in actigraphic sleep assessment. Sleep 2004;27:158-65

31. Morgenthaler T, Alessi C, Friedman L, et al. Practice parameters for the use of actigraphy in the assessment of sleep and sleep disorders: an update for 2007. Sleep 2007:30:519-29

32. Troosters $\mathbf{T}$, Gosselink R, Janssens $\mathbf{W}$, et al. Exercise training and pulmonary rehabilitation: new insights and remaining challenges. Eur Respir Rev 2010;19:24-9.

\section{Journal club}

\section{Mouse lung regeneration after H1N1}

This study demonstrates that following an ARDS-like syndrome in mice secondary to infection with a murine-adapted H1N1 virus, complete recovery follows the emergence of a population of stem cells bearing the differentiation markers of alveoli.

Contrary to the existing bleomycin-based models of pulmonary injury causing fibrosis, this murine H1N1 model sustains substantial airway damage and epithelial destruction, followed by viral clearing and histological recovery over several months. This represents a novel paradigm to investigate regenerative responses to infection.

Using molecular and immunohistochemical methods, the authors illustrate that following H1N1 infection, a cell population expressing p63, a known marker of stem cells in nasal and tracheal epithelia, emerges from distal bronchial epithelia in damaged lung and expands to form discrete 'pods' or islands of cells that concentrically surround distal airways. These pods assemble into novel alveoli-like structures bearing molecular markers and gene expression profiles of alveoli. Lineage tracing of these pods reveals a path originating in bronchiolar epithelium. Complete histological recovery including regeneration of alveoli-capillary networks follows over several months with no evidence of fibrosis. In parallel studies, the authors cultured comparable p63-positive stem cells derived from human distal airway epithelia, which similarly assembled into alveoli-like structures bearing specific molecular and genetic markers of alveolar and capillary development in vitro.

This work identifies a previously undescribed source of distal airway stem cells capable of regenerating damaged lung parenchyma following inflammation-induced lung injury, suggesting novel therapeutic approaches to currently non-reversible airway diseases. How the incipient alveoli-like structures integrate into existing airway structures remains to be seen.

Kumar PA, Hu Y, Yamamoto Y, et al. Distal airway stem cells yield alveoli in vitro and during lung regeneration following H1N1 influenza infection. Cell 2011;147:525-38.

\section{Imran Mahmud}

Correspondence to Dr Imran Mahmud, Oxford University Clinical Academic Graduate School, Hertford College, University of Oxford, OX1 3BW, UK; imran.mahmud@medsci.ox.ac.uk

Provenance and peer review Not commissioned; internally peer reviewed.

Published Online First 18 December 2012

Thorax 2012;67:734. doi:10.1136/thoraxjnl-2011-201438 